\title{
Induced Kozai Migration and Formation of Close-in Planets in Binaries
}

\author{
Genya Takeda, Ryosuke Kita, and Frederic A. Rasio
}

\author{
Dept. of Physics \& Astronomy, Northwestern University, \\ 2145 Sheridan Road, Evanston, IL USA \\ email: genya@u.northwestern.edu
}

\begin{abstract}
Many recent observational studies have concluded that planetary systems commonly exist in multiple-star systems. At least $\sim 20 \%$, and presumably a larger fraction, of the known extrasolar planetary systems are associated with one or more stellar companions. These stellar companions normally exist at large distances from the planetary systems (typical projected binary separations are $10^{2}-10^{4} \mathrm{AU}$ ) and are often faint (ranging from $\mathrm{F}$ to $\mathrm{T}$ spectral types). Yet, secular cyclic angular momentum exchange with these distant stellar companions can significantly alter the orbital configuration of the planets around the primaries. One of the most interesting and fairly common outcomes seen in numerical simulations is the opening of a large mutual inclination angle between the planetary orbits, forced by differential nodal precessions caused by the binary companion. The growth of the mutual inclination angle between planetary orbits induces additional large-amplitude eccentricity oscillations of the inner planet due to the quadrupole gravitational perturbation by the outer planet. This eccentricity oscillation may eventually result in the orbital decay of the inner planet through tidal friction, as previously proposed as Kozai migration or Kozai cycles with tidal friction (KCTF). This orbital decay mechanism induced by the binary perturbation and subsequent tidal dissipation may stand as an alternative formation channel for close-in extrasolar planets.
\end{abstract}

\section{Introduction}

As of June 2008 , at least $\sim 20 \%$ of the $\sim 240$ stars hosting planets are components of multiple-star systems (Desidera \& Barbieri 2007; Raghavan et al. 2006). Most of the binaries that harbor planets are very wide, with typical sky-projected separations ranging from $10^{2}$ to $10^{4} \mathrm{AU}$. The majority of the stellar companions to planetary systems are FGK-type stars, but there are also later-type companions, including $8 \mathrm{M}$-dwarfs and one T-type brown dwarf. These distant, and often faint, companions, however, may still play a significant role in altering planetary orbits around the primaries on a very long timescale. As first pointed out by Zucker \& Mazeh (2002), the two populations of planets, those around single stars and those around components of binary systems, seem to exhibit different orbital properties. The effect of secular binary perturbations is particularly discernible in the distribution of planets' orbital eccentricities, that are possibly excited by the Kozai mechanism ( $\S 2.1$ ). For example, a large fraction of planets with highly eccentric orbits $(e>0.7)$ are associated with stellar companions. Also, the population of planets in binaries shows a higher median eccentricity (0.25) compared to that of the planets around single stars $(0.20 \dagger)$. Another curious trend that was first noticed by Zucker \& Mazeh (2002) and still remains robust to date is the population of massive hot Jupiters $\left(m_{\mathrm{p}} \sin i>2 M_{\mathrm{J}}\right.$ and $P<100$ days $)$ found exclusively among binary systems

$\dagger$ observational biases seem to influence the observed eccentricity distribution only marginally; see for example Shen \& Turner (2008). 
(there are a few recent exceptions such as XO-3 b or HD 17156 b, for which the presence of stellar companions has not yet been ruled out).

Interestingly, secular eccentricity-pumping mechanisms such as the Kozai mechanism are closely related to the formation of close-in (possibly transiting) planets. In addition to the traditional planetary migration theory incorporating planet-disk interactions, there is a growing attention to an alternative mechanism involving circularization of initially eccentric orbits through tidal dissipation in the central stars. There are two major ways a planet can gain a sufficiently large orbital eccentricity that leads to the tidal circularization and orbital decay: mutual, impulsive gravitational scattering between planets upon dissipation of the nascent gas disk (Nagasawa et al. 2008), and adiabatic secular eccentricity growth induced by the Kozai mechanism (Wu \& Murray 2003; Fabrycky \& Tremaine 2007). The latter scenario, often called Kozai migration or Kozai cycles with tidal friction (KCTF), has been adopted for single-planet systems in binaries and produced models that are consistent with the observations, given certain assumptions in the initial conditions. In this study, we investigate the dynamical outcomes of multiple-planet systems secularly perturbed by stellar companions and find that the Kozai migration process is significantly enhanced with the presence of additional planetary companions.

\section{Secular Three-body Interactions}

We focus on the secular evolution of hierarchical four-body systems that consist of two planets around a component of a wide binary system. It would help to break up the problem into two secular three-body problems in order to provide analytical understandings of secular four-body interaction: (1) the secular evolution of two planets around the central star (nearly coplanar, not necessarily hierarchical), and the secular evolution of each planet due to the quadrupole perturbation from the binary (not necessarily coplanar, but highly hierarchical). The former has been formulated through the Laplace-Lagrange Secular Theory, and the latter through the Kozai mechanism.

\subsection{Secular Planet-Binary Interaction}

Binaries that contain planets are typically separated by more than $100 \mathrm{AU}$. In such wide binaries it is likely that the initial orbital angular momentum of the planet (i.e., the spinaxis orientation of the primary star) is not correlated to the orbital angular momentum of the binary (Hale 1994). Thus, distribution of the initial inclination of the planetary orbit with respect to the binary plane is expected to be isotropic. The Kozai mechanism takes its effect only when the initial relative inclination is greater than the critical Kozai angle $I_{\mathrm{Koz}}=39.2^{\circ}$. In such cases, the planet's eccentricity $(e)$, inclination $(I)$, the argument of pericenter $(\omega)$, and the longitude of ascending node $(\Omega)$, start to precess secularly all on similar timescales (called Kozai cycles; Kozai 1962). The orbital eccentricity of a planet oscillates, even if it is initially close to zero, with a nearly constant amplitude (see for example the red-dashed curve in Figure 1), that is to the lowest order a function only of the initial inclination $I_{0}$ (Holman et al. 1997):

$$
e_{\mathrm{max}} \approx \sqrt{1-5 / 3 \cos ^{2} I_{0}}
$$

The timescale of the eccentricity oscillation (and the evolution of other orbital elements) is determined as (Kiseleva et al. 1998)

$$
\tau_{\mathrm{Koz}} \approx \frac{2}{3 \pi} \frac{m_{0}+m_{\mathrm{p}}+m_{\mathrm{bin}}}{m_{\mathrm{bin}}}\left(1-e_{\mathrm{bin}}^{2}\right)^{3 / 2},
$$

where the subscripts 0 and bin denote the primary and the companion stars, respectively. 
During Kozai cycles, a planetary orbit is slowly and repeatedly torqued by the binary, keeping the orbital energy and thus the semi-major axis constant. A cumulative effect of the torque results in the secular apsidal precession of the planet, i.e., $\omega_{\mathrm{p}}$ precesses on the timescale $\tau_{\mathrm{Koz}}$. In a typical planetary system there are other sources of perturbations that also induce secular apsidal precession of the planet, such as the general-relativistic effect caused by the central star, or secular perturbations from other planetary companions. If any of these extra perturbations induces apsidal precessions on a timescale smaller than $\tau_{\mathrm{Koz}}$, the torque from the binary companion secularly averages out to zero, and the planetary orbit will remain nearly circular.

\subsection{Secular Mutual Interaction between Planets}

Analytical solutions for the long-term evolution of a pair of planets around a star due to their mutual gravitational interaction is formulated through the second-order LaplaceLagrange secular theory (L-L theory hereafter; Brouwer \& Clemence 1961; Murray \& Dermott 1999). The L-L theory also yields secular evolutionary timescales of the planets. The two eigenfrequencies $g_{+}$and $g_{-}$derived from the L-L theory correspond to the apsidal precession timescales, $\tau_{\omega, \pm} \approx 2 \pi / g_{ \pm}$. $\dagger$ This apsidal evolution timescale derived from the L-L theory can be compared to the Kozai timescale $\tau_{\mathrm{Koz}}$ (Eq. [2.2]) to determine whether the Kozai cycles are suppressed or not. If, for instance, a double-planet system in a binary has an initial configuration such that $\tau_{\omega, 2}<\tau_{\mathrm{Koz}, 2}$, then the Kozai cycles on the outer planet will be entirely suppressed, and the binary companion will play no significant role in the secular evolution of the outer planet.

The L-L theory also yields one eigenfrequency $f$ which scales with the coupling strength of the planetary ascending nodes $\ddagger$. This also translates into the nodal precession timescale of a double-planet system as $\tau_{\Omega} \approx 2 \pi / f$.

\section{Non-rigid Evolution of Planetary Orbits in Binaries}

Newly formed planets emerging from the circumstellar gas disk are expected to orbit in a common plane (along the stellar equator). However, in the quadrupolar gravitational potential of the binary, this coplanarity can be broken as the planets evolve (Takeda et al. 2008). If the nodal coupling of the planets (scales with $\tau_{\Omega}$ ) is strong compared to the nodal perturbation caused by the binary companion (scales with $2 \tau_{\mathrm{Koz}}$ ), planetary orbits can evolve rigidly, maintaining near-coplanarity. On the other hand, if the nodal coupling between the planets is weak compared to the nodal perturbation introduced by the stellar companion, a large mutual inclination angle starts to grow between the planetary orbits, thereby inducing Kozai cycles to the orbit of the inner planet.

In Figure 1, an initially zero mutual inclination $\left(I_{12}\right)$ between the planetary orbits grows periodically to as large as $\sim 60^{\circ}$. As soon as the mutual inclination between the planets reaches $40^{\circ}$ at $t \sim 2 \mathrm{Myr}$, the inner planet's orbital eccentricity $e_{1}$ starts to oscillate chaotically, reaching as high as $\sim 0.95$. This growth of $I_{12}$ is solely due to the differential nodal precession of the planetary orbits introduced by the stellar companion (Takeda et al. 2008). It is almost independent of how inclined initially the planetary orbits are with respect to the binary plane, unlike the three-body Kozai mechanism. In

$\dagger$ In certain limits the apsidal evolution is described as a nonlinear combination of multiple eigenmodes, and this simple relation between the eigenfrequency and the apsidal precession frequency does not hold. See Takeda et al. (2008).

$\ddagger$ If an additional quadrupolar potential such as stellar oblateness is introduced, there will be two eigenfrequencies in the $I-\Omega$ space. See Murray \& Dermott (1999). 


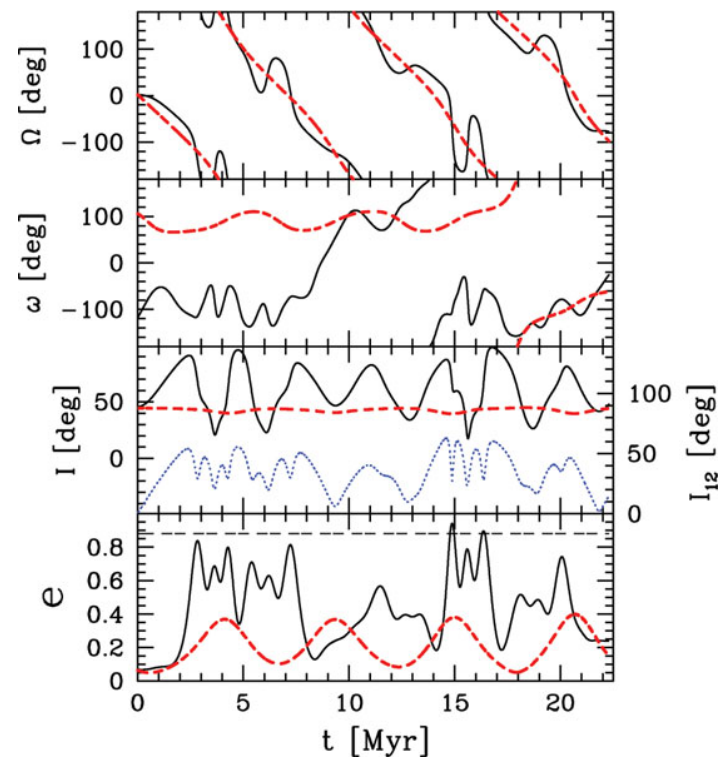

Figure 1. Numerically integrated evolution of a non-rigid double-planet system in a binary. The system evolved from the following initial conditions: for the inner planet (solid black curve), $m_{1}=0.02 M_{\mathrm{J}}, a_{1}=0.9 \mathrm{AU}$ and $I_{1}=45^{\circ}$; for the outer planet (dashed red curve), $m_{2}=0.6 M_{\mathrm{J}}$, $a_{2}=12.7 \mathrm{AU}$ and $I_{2}=45^{\circ}$; and for the binary companion, $m_{\mathrm{bin}}=0.7 M_{\odot}, a_{\mathrm{bin}}=803 \mathrm{AU}$ and $e_{\mathrm{bin}}=0.78$. The binary orbit remains at $I_{\mathrm{bin}}=0^{\circ}$. The dotted blue curve represents the relative inclination angle $I_{12}$ between the two planets (scale shown on the right axis). At around $t=14.5 \mathrm{Myr}$, the inner planet's pericenter enters inside $0.1 \mathrm{AU}$ of the central star (horizontal dashed line in the bottom panel) and remains there for $\sim 2 \times 10^{5} \mathrm{yr}$.

four-body systems, the orbital eccentricity of the inner planet can still grow to a very large value even if the planet's initial inclination is below the critical Kozai angle.

In an opposite case in which the nodal coupling of the planetary orbits is sufficiently strong compared to the nodal precession of the outer planet induced by the stellar companion, planetary orbits secularly rotate together, remaining on a common plane. In such dynamically-rigid configuration, $I_{12}$ and $e_{1}$ remain small throughout the evolution.

\section{Kozai Migration and Formation of Close-in Planets in Binaries}

In dynamically non-rigid systems the inner planet's eccentricity secularly oscillates due to the perturbation from the outer planet. During these Kozai cycles, without any dissipative mechanism the semi-major axis $a_{1}$ of the inner planet remains constant throughout the evolution. This means that at each eccentricity maximum, the planet's pericenter distance $q_{1}=a_{1}\left(1-e_{1}\right)$ reduces greatly. It is known that in the regime where $q_{1} \lesssim 0.1 \mathrm{AU}$, strong tidal interaction with the central star leads to damping of the orbital eccentricity and the semi-major axis, and the planetary orbit migrates toward the central star until it eventually halts at a tight, nearly circular orbit (Kiseleva et al. 1998; Wu \& Murray 2003; Fabrycky \& Tremaine 2007). For example, in the example in Figure 1 the inner planet is expected to start decaying its orbit around $t \approx 15 \mathrm{Myr}$ if tidal dissipation is included in the calculation.

This so-called Kozai migration scenario has been adopted to produce some dynamical models that are consistent with observations (e.g., HD 80606 b; Wu \& Murray 2003), 
given relatively fine-tuned initial conditions. The difficulty is to achieve such a small pericenter distance during the Kozai cycles that the tidal dissipation significantly drains the orbital energy from the planet. Thus these dynamical models require very large initial relative inclination angles, almost $90^{\circ}$. Such a large angle is not unrealistic, but not likely to be applicable to many systems.

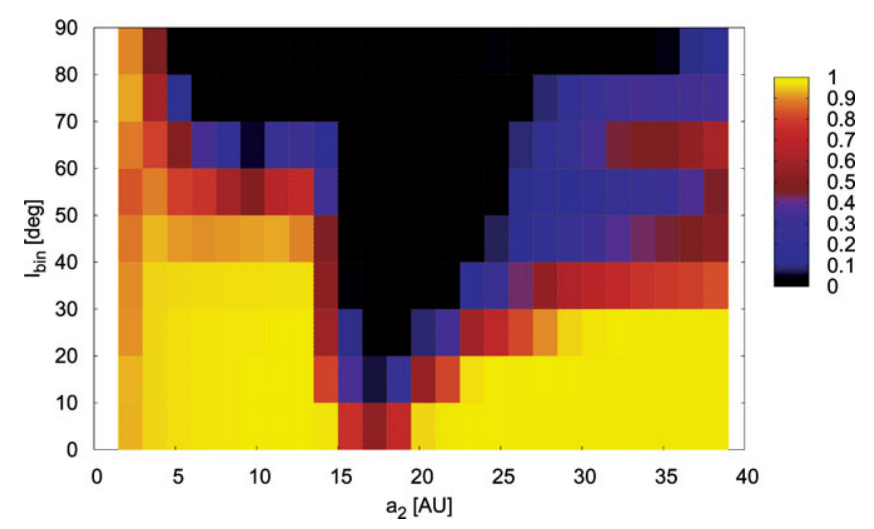

Figure 2. The minimum pericenter distance in $\mathrm{AU}$ reached by the inner planet within $500 \mathrm{Myr}$ of numerical integrations with different sets of $I_{\text {bin }}$ and $a_{2}$. Other orbital parameters and masses are fixed as $m_{0}=m_{\text {bin }}=1.0 M_{\odot}, m_{1}=0.1 M_{\mathrm{J}}, m_{2}=1.0 M_{\mathrm{J}}, a_{1}=1 \mathrm{AU}, a_{\text {bin }}=750 \mathrm{AU}$, and $e_{\text {bin }}=0.5$. The darker-shaded area corresponds to the smaller pericenter distance achieved by the inner planet. Planets that reach pericenter approach smaller than $\sim 0.1 \mathrm{AU}$ are subject to tidal dissipation and expected to start migrating toward the central star.

However, as explained in the previous section, in a more realistic planetary system containing more than one planet, such a tight requirement on the initial inclination angle is no longer necessary. Figure 2 shows the distribution of minimum pericenter distances achieved by the inner planet during $500 \mathrm{Myr}$ of numerical integration with different sets of initial $a_{2}$ and $I_{\text {bin }}$. The parameter space is divided into two evolutionary classes depending on the location of the outer planet; in the region where $a_{2} \lesssim 15 \mathrm{AU}$, the planetary system is dynamically rigid, and the inner planet's eccentricity remains small (pericenter remains large). The only exceptions in this region are when the planetary orbits are inclined from the binary by more than $\sim 60^{\circ}$, in which case close encounter or possible orbital crossing between the planets induced by the large-amplitude Kozai cycles of the outer planet impulsively excites $e_{1}$ to large values. If, on the other hand, $a_{2} \gtrsim 15$ AU initially, then the system is dynamically non-rigid, and even if the planetary orbits and the binary plane are initially nearly coplanar, additional Kozai cycles will significantly excite the orbital eccentricity of the inner planet.

Such a numerical coverage of the $a_{2}-I_{\text {bin }}$ space shows similar morphology even if different initial binary parameters and planetary masses are selected; there is always a critical orbital separation $a_{2, \text { crit }}$ that separates dynamically-rigid and non-rigid evolutions. As illustrated in Figure 2, the formation of close-in planets through tidal circularization of eccentric orbits should be particularly efficient if the outer planet is initially located beyond $a_{2, \text { crit }}$. This boundary $a_{2 \text {,crit }}$ can be analytically determined by simple formulae, given the initial orbital parameters of the system (Takeda et al. 2008). 


\section{Properties of Close-in Planets Formed via Enhanced Kozai Migration}

As demonstrated in the previous chapter, a stellar companion can effectively stir up planetary systems from a large distance and excite the eccentricity of the innermost planet by propagating its perturbation inward through planets on a reasonable timescale. Once the planet's eccentricity becomes sufficiently large and the pericenter distance sufficiently small, the planet begins to migrate toward the central star until it settles on a tight, circular orbit. It is possible to identify close-in planets in binaries that formed via such an "induced Kozai migration" scenario from their final orbital properties.

The leading hypothesis for the planet migration mechanism involves the loss of angular momentum through interaction with the protoplanetary gas disk (e.g., Goldreich \& Sari 2003). If the planet-disk interaction is the common migration channel for extrasolar planets, the majority of the close-in planets are expected to be found on orbits along the stellar equator. On the other hand, if a planet migrates via the combined effect of the Kozai cycles and tidal dissipation, the angular momentum of the final planetary orbit should be largely misaligned with respect to the stellar spin axis since the planet's inclination evolves during the Kozai cycles $\dagger$. The sky-projected angle $\lambda$ of spin-orbit misalignment is now measurable for transiting planets through the Rossiter-McLaughlin (RM) effect (e.g., Winn et al. 2005). Among the first 8 extrasolar planets with available $\mathrm{RM}$ measurements, one of them shows a possible large spin-orbit misalignment; XO-3b $\left(\lambda=70^{\circ} \pm 15^{\circ}\right.$; Hébrard et al. 2008). Although this result still need to be verified by follow-up observations, once the spin-orbit misalignment is confirmed, it will be the first strong candidate transiting systems that formed through Kozai migration.

Another curious observational trend is the presence of massive close-in planets found predominantly among binaries. A possible indication of this trend is that massive planets somehow do not migrate efficiently through planet-disk interaction. If that is true, then Kozai migration triggered by binary perturbations would stand as a strong alternative to form close-in planets with a wide range of masses. Another possible common migration channel, planet-planet scattering during the early stage of planet formation (Chatterjee et al. 2008; Nagasawa et al. 2008), also faces a significant challenge in migrating massive planets into tight orbits because it is usually the lighter planets that get scattered inward. The Kozai mechanism combined with tidal damping so far is the only process that does not depend strongly on planetary masses, consistent with the exclusive presence of massive ones in binaries. This is another reason to suspect that the two transiting planets HD 17156b $\left(m_{\mathrm{p}}=3.09_{-0.17}^{+0.22} M_{\mathrm{J}} ;\right.$ Gillon et al. 2008$)$ and XO-3b $\left(m_{\mathrm{p}}=11-\right.$ $13 M_{\mathrm{J}}$; Johns-Krull et al. 2008; Winn et al. 2008) are the outcomes of Kozai migration, as both planets are fairly massive.

The orbital migration process proposed in this study requires at least four or more bodies in a system, including two stars and two (or more) planets. Alternatively, for a handful of known close-in single planets in binaries, detections of secondary planets will significantly constrain the dynamical origin of those systems. Note that the induced Kozai migration is more common in hierarchical planetary systems where the second planet orbits beyond a critical radius $a_{2, \text { crit }}$ such that the planetary orbits evolve nonrigidly. Unfortunately, detection of additional planets beyond a few AU will take several years or decades of continuous radial-velocity monitoring. Once the presence of additional

$\dagger$ Theoretically, orbital inclination can also be damped toward the stellar equator through tidal dissipation. However, it usually takes longer than the estimated age of the planetary system; see Hut (1981); Winn et al. (2005). 
planetary companions in those systems are ruled in or out, however, the dynamical history of known close-in planets can be significantly constrained.

\section{Acknowledgements}

This work was supported by NSF Grant AST-0507727.

\section{References}

Brouwer, D. \& Clemence, G. M. 1961, Methods of celestial mechanics (New York: Academic Press, 1961)

Chatterjee, S., Ford, E. B., \& Rasio, F. A. 2008, ApJ

Desidera, S. \& Barbieri, M. 2007, A\&BA, 462, 345

Fabrycky, D. \& Tremaine, S. 2007, ApJ, 669, 1298

Gillon, M., Triaud, A. H. M. J., Mayor, M., Queloz, D., Udry, S., \& North, P. 2008, A\& A, 485, 871

Goldreich, P. \& Sari, R. 2003, ApJ, 585, 1024

Hale, A. 1994, AJ, 107, 306

Hebrard, G., Bouchy, F., Pont, F., et al. 2008, A\&A, 466, 763

Holman, M., Touma, J., \& Tremaine, S. 1997, Nature, 386, 254

Hut, P. 1981, A\&A, 99, 126

Johns-Krull, C. M., McCullough, et al., 2008, ApJ, 677, 657

Kiseleva, L. G., Eggleton, P. P., \& Mikkola, S. 1998, MNRAS, 300, 292

Kozai, Y. 1962, AJ, 67, 591

Murray, C. D. \& Dermott, S. F. 1999, Solar system dynamics

Nagasawa, M., Ida, S., \& Bessho, T. 2008, ApJ, 678, 498

Raghavan, D., Henry, T. J., Mason, B. D., Subasavage, J. P., Jao, W.-C., Beaulieu, T. D., \& Hambly, N. C. 2006, ApJ, 646, 523

Shen, Y. \& Turner, E. L. 2008, arXiv:0806.0032v1 [astro-ph]

Takeda, G., Kita, R., \& Rasio, F. A. 2008, ApJ, in press

Winn, J. N., Holman, M. J., Torres, G., McCullough, P., et al. 2008, arXiv:0804.4475

Winn, J. N., Noyes, R. W., Holman, M. J., Charbonneau, D., Ohta, Y., Taruya, A., Suto, Y., Narita, N., Turner, E. L., Johnson, J. A., Marcy, G. W., Butler, R. P., \& Vogt, S. S. 2005, ApJ, 631, 1215

Wu, Y. \& Murray, N. 2003, ApJ, 589, 605

Zucker, S. \& Mazeh, T. 2002, ApJL, 568, L113 